\title{
Read Me
}

File "1_Aphid_abundance_cereal"

This file contains data on the number and species of aphids sampled in 2018 and 2019 in cereal and mixed crops. Each line corresponds to a cereal plant sampled, on which the number/species of aphid was determined. For method, please read the Material and methods of the corresponding article.

Year: year of the sampling (2018/2019).

Week: number of the week during which the sampled was collected (week one (W1) is the week of January 1rst).

Period: the growing season was divided in three periods: elongation (P1), heading (P2) and ripening (P3). See Table 1 for specific sampling dates.

Field: the identification of the field in which the sampling is performed.

Type: Crop type, i.e. cereal crop ("Cereal") or mixed crops ("Mixed").

Plant_ID: identification of the plant sampled (50 plants per field are sampled, see Material and methods).

S_avenae: number of Sitobion avenae sampled on the plant

R_padi: number of Rhopalosiphum padi sampled on the plant

M_dirhodum: number of Metopolophium dirhodum sampled on the plant

Mummy: number of aphid mummies sampled on the plant

File "2_Parasitism_rate_cereal”

This file contains data used to calculate cereal aphid parasitism rate in 2018 and 2019 in cereal and mixed crops. Each line corresponds to a cereal plant sampled, on which the number/species of aphid was determined, as well as the number of aphid mummies. For method, please read the Material and methods of the corresponding article.

Year: year of the sampling (2018/2019).

Week: number of the week during which the sampled was collected (week one (W1) is the week of January 1 rst).

Period: the growing season was divided in three periods: elongation (P1), heading (P2) and ripening (P3). See Table 1 for specific sampling dates.

Field: the identification of the field in which the sampling is performed.

Type: Crop type, i.e. cereal crop ("Cereal") or mixed crops ("Mixed").

Plant_ID: identification of the plant sampled (30 plants per field are sampled in 2019, see Material and methods). 
S_avenae: number of Sitobion avenae sampled on the plant

R_padi: number of Rhopalosiphum padi sampled on the plant

M_dirhodum: number of Metopolophium dirhodum sampled on the plant

Mummy: number of aphid mummies sampled on the plant

\section{File "3_Foodweb_cereal_primary_parasitoids"}

This file contains data used to calculate cereal aphid-primary parasitoid foodweb indices, as well as the relative abundances of each parasitoid species in 2018 and 2019 in cereal and mixed crops. Each line corresponds to an aphid mummy that was identified, and the parasitoid species emerging from this mummy. For method, please read the Material and methods of the corresponding article. Year: year of the sampling (2018/2019).

Week: number of the week during which the sampled was collected (week one (W1) is the week of January 1 rst).

Period: the growing season was divided in three periods: elongation (P1), heading (P2) and ripening (P3). See Table 1 for specific sampling dates.

Field: the identification of the field in which the sampling is performed.

Type: Crop type, i.e. cereal crop ("Cereal") or mixed crops ("Mixed").

Aphid_sp: aphid species of the mummy collected (M. dirhodum, S. avenae or R. padi).

Parasitoid_sp: primary parasitoid emerging from the mummy (A. rhopalosiphi, A. ervi, A. matricariae, A. avenae, P. volucre, E. plagiator).

A_ervi: binary (0/1): presence or absence of the parasitoid A. ervi

A_avenae: binary (0/1): presence or absence of the parasitoid $A$. avenae

A_rhopa: binary (0/1): presence or absence of the parasitoid $A$. rhopalosiphi

A_matricariae: binary (0/1): presence or absence of the parasitoid $A$. matricariae

P_volucre: binary (0/1): presence or absence of the parasitoid $P$. volucre

E_plagiator: binary (0/1): presence or absence of the parasitoid E. plagiator

\section{File "4_Foodweb_hyperparasitoid"}

This file contains data used to calculate cereal aphid-hyperparasitoid foodweb indices, as well as hyperparasitism rate in 2018 and 2019 in cereal and mixed crops. Each line corresponds to an aphid mummy that was identified, and the parasitoid or hyperparasitoid species emerging from this mummy. In 2018, no information on the hyperparasitoid genus was obtained ("NA"). In this data set, the alternative hosts ( $A$. pisum) are included. For method, please read the Material and methods of the corresponding article.

Year: year of the sampling (2018/2019). 
Week: number of the week during which the sampled was collected (week one (W1) is the week of January 1 rst).

Period: the growing season was divided in three periods: elongation (P1), heading (P2) and ripening (P3). See Table 1 for specific sampling dates.

Field: the identification of the field in which the sampling is performed.

Type: Crop type, i.e. cereal crop (“Cereal”) or mixed crops (“Mixed").

Aphid_sp: aphid species of the mummy collected (A. pisum, Aphis app., M. dirhodum, S. avenae or R. padi).

Parasitoid_sp: primary parasitoid emerging from the mummy (A. rhopalosiphi, A. ervi, A. matricariae, A. avenae, P. volucre, E. plagiator, A. banksae, A. eadyi, A. smithi, Lysiphlebus), or "na" if no primary parasitoid emerged.

Hyper: Genus of the hyperparasitoid emerging from the mummy (Alloxysta, Asaphes, Phaenoglyphis, Coruna, Dendrocerus), or "na" if no hyperparasitoid emerged.

Prop_hyper: binary (0/1): 0 for a primary parasitoid/1 for a hyperparasitoid.

\section{File "5_Foodweb_alternative_hosts"}

This file contains foodweb data on aphid species found on the leguminous plants (Acyrthosiphon pisum) and their emerging parasitoids, in 2018 and 2019. Each line corresponds to an aphid mummy that was identified, and the parasitoid emerging from this mummy. For method, please read the Material and methods of the corresponding article.

Year: year of the sampling (2018/2019).

Week: number of the week during which the sampled was collected (week one (W1) is the week of January 1rst).

Period: the growing season was divided in three periods: elongation (P1), heading (P2) and ripening (P3). See Table 1 for specific sampling dates.

Field: the identification of the field in which the sampling is performed.

Type: Crop type, i.e. cereal crop (“Cereal”) or mixed crops (“Mixed").

Aphid_sp: aphid species of the mummy collected (A. pisum).

Parasitoid_sp: primary parasitoid emerging from the mummy (A. rhopalosiphi, A. ervi, A. matricariae, A. avenae, P. volucre, A. banksae, A. eadyi, A. smithi).

A_ervi: binary (0/1): presence or absence of the parasitoid A. ervi

A_avenae: binary (0/1): presence or absence of the parasitoid $A$. avenae

A_rhopa: binary (0/1): presence or absence of the parasitoid A. rhopalosiphi

A_matricariae: binary (0/1): presence or absence of the parasitoid A. matricariae

P_volucre: binary (0/1): presence or absence of the parasitoid $P$. volucre 
A_banksae: binary (0/1): presence or absence of the parasitoid A. banksae

A_eadyi: binary (0/1): presence or absence of the parasitoid A. eadyi

A_smithi: binary (0/1): presence or absence of the parasitoid $A$. smithi

File "6_Supplementary_sizes"

This file contains data used for Supporting information S3. Measurements of aphid and parasitoid sizes were performed in individuals collected in 2019. For method, please read the Material and methods in S3 of the corresponding article.

Week: number of the week during which the sampled was collected (week one (W1) is the week of January 1 rst).

Period: the growing season was divided in three periods: elongation (P1), heading (P2) and ripening (P3). See Table 1 for specific sampling dates.

Type: Crop type, i.e. cereal crop ("Cereal") or mixed crops ("Mixed").

Aphid_sp: aphid species of the mummy collected (A. pisum, S.avenae).

Parasitoid_sp: primary parasitoid emerging from the mummy (A. rhopalosiphi, A. ervi).

Aphid_size: hind tibia length $( \pm 0.01 \mathrm{~mm})$ of the aphid mummy.

Parasitoid_size: hind tibia length $( \pm 0.01 \mathrm{~mm})$ of the emerging parasitoid. 